\title{
Homogenization of a parabolic equation in perforated domain with Neumann boundary condition
}

\author{
A K NANDAKUMARAN and M RAJESH* \\ Department of Mathematics, Indian Institute of Science, Bangalore 560 012, India \\ *Corresponding author \\ E-mail: nands@math.iisc.ernet.in; rajesh@math.iisc.ernet.in
}

Abstract. In this article, we study the homogenization of the family of parabolic equations over periodically perforated domains

$$
\begin{aligned}
\partial_{t} b\left(\frac{x}{\varepsilon}, u_{\varepsilon}\right)-\operatorname{div} a\left(\frac{x}{\varepsilon}, u_{\varepsilon}, \nabla u_{\varepsilon}\right) & =f(x, t) \text { in } \Omega_{\varepsilon} \times(0, T), \\
a\left(\frac{x}{\varepsilon}, u_{\varepsilon}, \nabla u_{\varepsilon}\right) \cdot v_{\varepsilon} & =0 \text { on } \partial S_{\varepsilon} \times(0, T), \\
u_{\varepsilon} & =0 \text { on } \partial \Omega \times(0, T), \\
u_{\varepsilon}(x, 0) & =u_{0}(x) \text { in } \Omega_{\varepsilon} .
\end{aligned}
$$

Here, $\Omega_{\varepsilon}=\Omega \backslash S_{\varepsilon}$ is a periodically perforated domain. We obtain the homogenized equation and corrector results. The homogenization of the equations on a fixed domain was studied by the authors [15]. The homogenization for a fixed domain and $b\left(\frac{x}{\varepsilon}, u_{\varepsilon}\right) \equiv b\left(u_{\varepsilon}\right)$ has been done by Jian [11].

Keywords. Homogenization; perforated domain; two-scale convergence; correctors.

\section{Introduction}

Let $\Omega$ be a bounded domain in $\mathbb{R}^{n}$ with smooth boundary $\partial \Omega$. Let $T>0$ be a constant and let $\varepsilon>0$ be a small parameter which eventually tends to zero. Let $Y=(0,1)^{n}$ and $S$ (closed) $\subset Y$. We define a periodically perforated domain $\Omega_{\varepsilon}$ as follows: First define

$$
\begin{aligned}
& I_{\varepsilon}=\left\{k \in \mathbb{Z}^{n}: \varepsilon(k+S) \subset \Omega\right\} \text { and, } \\
& S_{\varepsilon}=\bigcup_{k \in I_{\varepsilon}} \varepsilon(k+S) .
\end{aligned}
$$

Set

$$
\Omega_{\varepsilon}=\Omega \backslash S_{\varepsilon}
$$

We assume that, (i) $\partial \Omega_{\varepsilon}$ is smooth and, (ii) $\Omega_{\varepsilon}$ is connected. Note that in the definition of the holes $S_{\varepsilon}$ we have taken them to have no intersection with the external boundary $\partial \Omega$. This is only done to avoid technicalities and the results of our paper will remain valid without this assumption (cf. [2] for a refinement of our arguments). 
We consider the following family of equations with Neumann conditions on the boundary of the holes

$$
\begin{aligned}
\partial_{t} b\left(\frac{x}{\varepsilon}, u_{\varepsilon}\right)-\operatorname{div} a\left(\frac{x}{\varepsilon}, u_{\varepsilon}, \nabla u_{\varepsilon}\right) & =f(x, t) \text { in } \Omega_{\varepsilon} \times(0, T), \\
a\left(\frac{x}{\varepsilon}, u_{\varepsilon}, \nabla u_{\varepsilon}\right) \cdot v_{\varepsilon} & =0 \text { on } \partial S_{\varepsilon} \times(0, T), \\
u_{\varepsilon} & =0 \text { on } \partial \Omega \times(0, T), \\
u_{\varepsilon}(x, 0) & =u_{0}(x) \text { in } \Omega_{\varepsilon},
\end{aligned}
$$

where $u_{0}$ is a given function on $\Omega$ and $f$ is a given function on $\Omega \times(0, T)$. For given $\varepsilon$, the Cauchy problem (1.4) will also be denoted by $\left(P_{\varepsilon}\right)$. It is known that under suitable assumptions on $a$ and $b$ (cf. assumptions (A1)-(A4) below), that the problem $\left(P_{\varepsilon}\right)$ has a solution $u_{\varepsilon}$. Our aim in this paper is to study the homogenization of the equations $\left(P_{\varepsilon}\right)$ as $\varepsilon \rightarrow 0$ i.e. to study the limiting behaviour of $u_{\varepsilon}$ as $\varepsilon \rightarrow 0$ and obtain the limiting equation satisfied by the limit. The theory of homogenization is a well-developed area of research $[5,12]$ and we are not going into the details of this theory. The case when $b$ is linear (i.e. $b(y, s)=s$ ) has been studied quite widely $[4,6-8,10,16]$. When $b$ is not linear, the homogenization of the equation in a fixed domain was studied by Jian [11] for $b(y, s) \equiv b(s)$ and in the general case, by the authors [15].

Assuming that $\tilde{u_{\varepsilon}}$ are a priori bounded in $L^{\infty}(\Omega \times(0, T)$ ) (where $\sim$ denotes the extension of $u_{\varepsilon}$ by zero in the holes), we show that $\tilde{u_{\varepsilon}}$ converges, for a subsequence, to a solution of the homogenized problem

$$
\begin{array}{rlrl}
\partial_{t} b^{*}(u)-\operatorname{div} A(u, \nabla u) & =m^{*} f(x, t) \quad \text { in } \Omega \times(0, T), \\
u & =0 \quad & \text { on } \partial \Omega \times(0, T), \\
u(x, 0) & =0 \quad & \text { in } \Omega,
\end{array}
$$

where $b^{*}$ and $A$ have been identified (cf. eqs (2.13)-(2.15)); $m^{*}$ denotes the volume fraction of $Y \backslash S$ in $Y$, i.e. $m^{*}=|Y \backslash S|$, the Lebesgue measure of $Y \backslash S$. We also improve the weak convergence of $\tilde{u_{\varepsilon}}$ by obtaining correctors. Such results are very useful in obtaining numerical approximations and they form an important aspect of homogenization.

The layout of the paper is as follows: In $\S 2$, we give the weak formulation for the problem $\left(P_{\varepsilon}\right)$. Then we state our main results viz. Theorems 2.4 and 2.6. In $\S 3$, we sketch some preliminary results and recall facts about the two-scale convergence method. Finally, in $\S 4$, we prove our main results in the framework of two-scale convergence.

\section{Assumptions and main results}

For $p>1, p^{*}$ will denote the conjugate exponent $p /(p-1)$. Define the space $V_{\varepsilon} \doteq\{v \in$ $W^{1, p}\left(\Omega_{\varepsilon}\right): v=0$ on $\left.\partial \Omega\right\} ; V_{\varepsilon}^{*}$ is its dual and $E_{\varepsilon} \doteq L^{p}\left(0, T ; V_{\varepsilon}\right)$. We define $u_{\varepsilon} \in E_{\varepsilon}$ to be a weak solution of $\left(P_{\varepsilon}\right)$ if it satisfies:

$$
b\left(\frac{x}{\varepsilon}, u_{\varepsilon}\right) \in L^{\infty}\left(0, T ; L^{1}\left(\Omega_{\varepsilon}\right)\right), \partial_{t} b\left(\frac{x}{\varepsilon}, u_{\varepsilon}\right) \in L^{p^{*}}\left(0, T ; V_{\varepsilon}^{*}\right),
$$

that is

$$
\begin{array}{r}
\int_{0}^{T}\left\langle\partial_{t} b\left(\frac{x}{\varepsilon}, u_{\varepsilon}\right), \xi(x, t)\right\rangle_{\varepsilon} \mathrm{d} t+\int_{0}^{T} \int_{\Omega_{\varepsilon}}\left\langle b\left(\frac{x}{\varepsilon}, u_{\varepsilon}\right)-b\left(\frac{x}{\varepsilon}, u_{0}\right)\right\rangle \\
\partial_{t} \xi \mathrm{d} x \mathrm{~d} t=0
\end{array}
$$


for all $\xi \in E_{\varepsilon} \cap W^{1,1}\left(0, T ; L^{\infty}\left(\Omega_{\varepsilon}\right)\right)$ with $\xi(T)=0$; and,

$$
\begin{aligned}
\int_{0}^{T}\left\langle\partial_{t} b\left(\frac{x}{\varepsilon}, u_{\varepsilon}\right), \xi(x, t)\right\rangle_{\varepsilon} \mathrm{d} t & +\int_{0}^{T} \int_{\Omega_{\varepsilon}} a\left(\frac{x}{\varepsilon}, u_{\varepsilon}, \nabla u_{\varepsilon}\right) \cdot \nabla \xi(x, t) \mathrm{d} x \mathrm{~d} t \\
& =\int_{0}^{T} \int_{\Omega_{\varepsilon}} f(x, t) \xi(x, t) \mathrm{d} x \mathrm{~d} t
\end{aligned}
$$

for all $\xi \in E_{\varepsilon}$. Here $\langle., .\rangle_{\varepsilon}$ denotes the duality bracket with respect to $E_{\varepsilon}^{*}, E_{\varepsilon}$.

Note: For any $\xi \in L^{p}\left(0, T ; W_{0}^{1, p}(\Omega)\right)$, its restriction to $\Omega_{\varepsilon}$ can be used as a test function in (2.3) for the problem $\left(P_{\varepsilon}\right)$.

We make the following assumptions on $a$ and $b$ :

(A1) The function $b(y, s)$ is continuous in $y$ and $s, Y$-periodic in $y$ and non-decreasing in $s$ and $b(y, 0)=0$.

(A2) There exists a constant $\theta>0$ such that for every $\delta$ and $R$ with $0<\delta<R$, there exists $C(\delta, R)>0$ such that

$$
\left|b\left(y, s_{1}\right)-b\left(y, s_{2}\right)\right|>C(\delta, R)\left|s_{1}-s_{2}\right|^{\theta}
$$

for all $y \in Y$ and $s_{1}, s_{2} \in[-R, R]$ with $\delta<\left|s_{1}\right|$.

Remark 2.1. The prototype for $b$ is a function of the form $c(y)|s|^{k} \operatorname{sgn}(s)$ for some positive real number $k$ and continuous and $Y$-periodic function, $c($.$) , which is positive on Y$.

(A3) The mapping $(y, \mu, \lambda) \mapsto a(y, \mu, \lambda)$ defined from $\mathbb{R}^{n} \times \mathbb{R} \times \mathbb{R}^{n}$ to $\mathbb{R}^{n}$ is measurable and $Y$-periodic in $y$ and continuous in $(\mu, \lambda)$. Further, it is assumed that there exists positive constants $\alpha, r$ such that

$$
\begin{aligned}
& a(y, \mu, \lambda) . \lambda \geq \alpha|\lambda|^{p}, \\
& \left(a\left(y, \mu, \lambda_{1}\right)-a\left(y, \mu, \lambda_{2}\right)\right)\left(\lambda_{1}-\lambda_{2}\right)>0, \quad \forall \lambda_{1} \neq \lambda_{2} \\
& |a(y, \mu, \lambda)| \leq \alpha^{-1}\left(1+|\mu|^{p-1}+|\lambda|^{p-1}\right), \\
& \left|a\left(y, \mu_{1}, \lambda\right)-a\left(y, \mu_{2}, \lambda\right)\right| \leq \alpha^{-1}\left|\mu_{1}-\mu_{2}\right|^{r}\left(1+\left|\mu_{1}\right|^{p-1-r}\right. \\
& \left.\quad+\left|\mu_{2}\right|^{p-1-r}+|\lambda|^{p-1-r}\right) .
\end{aligned}
$$

(A4) We assume that the data, $f \in L^{\infty}(\Omega \times T)$. Under the above assumptions, it is known that $\left(P_{\varepsilon}\right)$ admits a solution $u_{\varepsilon}$ (cf. [3]).

Remark 2.2. From hereon, we make the following simplifying assumption to reduce the technicalities in the proof of the homogenization, viz. that $a(y, \mu, \lambda)$ is of the form $a(y, \lambda)$. The same results can be carried over for general $a$ (cf. see [11,15] for suitable modifications).

(A5) The strong monotonicity condition;

$$
\left(a\left(y, \lambda_{1}\right)-a\left(y, \lambda_{2}\right)\right) .\left(\lambda_{1}-\lambda_{2}\right) \geq \alpha\left|\lambda_{1}-\lambda_{2}\right|^{p} .
$$


Remark 2.3. We use the condition (A5) only for obtaining the corrector result. It will not be required for identifying the homogenized problem.

We now state our main theorems.

Theorem 2.4. Let $u_{\varepsilon}$ be a family of solutions of $\left(P_{\varepsilon}\right)$. Assume that there is a constant $C>0$, such that

$$
\sup _{\varepsilon}\left\|u_{\varepsilon}\right\|_{L^{\infty}\left(\Omega_{\varepsilon} \times(0, T)\right)} \leq C .
$$

Then there exists a subsequence of $\varepsilon$, still denoted by $\varepsilon$, such that for all $q$ with $0<q<\infty$, we have

$$
\tilde{u_{\varepsilon}} \rightarrow u \text { strongly in } L^{q}\left(\Omega_{T}\right)
$$

and $u$ solves,

$$
\begin{aligned}
\partial_{t} b^{*}(u)-\operatorname{div} A(\nabla u) & =m^{*} f \text { in } \Omega_{T}, \\
u & =0 \text { on } \partial \Omega \times(0, T), \\
u(x, 0) & =u_{0} \text { in } \Omega,
\end{aligned}
$$

where $b^{*}$ and $A$ are defined below by (2.13)-(2.15), $m^{*}=|Y \backslash S|$ and $\Omega_{T}$ denotes the set $\Omega \times(0, T)$.

The functions $b^{*}$ and $A$ are defined through,

$$
\begin{array}{r}
b^{*}(s)=\int_{Y \backslash S} b(y, s) \mathrm{d} y, \\
A(\lambda)=\int_{Y \backslash S} a\left(y, \lambda+\nabla \Phi_{\lambda}(y)\right) \mathrm{d} y,
\end{array}
$$

where $\lambda \in \mathbb{R}^{n}$ and $\Phi_{\lambda} \in W_{\text {per }}^{1, p}(Y)$ solves the periodic boundary value problem

$$
\int_{Y \backslash S} a\left(y, \lambda+\nabla \Phi_{\lambda}(y)\right) . \nabla \phi(y) \mathrm{d} y=0
$$

for all $\phi \in W_{\text {per }}^{1, p}(Y)$. See [9] for further details about eq. (2.15) and for the properties of $A$.

Remark 2.5. The assumption (2.10) is true in special cases (see [13]) and it is reasonable on physical grounds (see [11]).

We see that by extending $u_{\varepsilon}$ by zero in the holes we do not get functions in $L^{p}\left(0, T ; W_{0}^{1, p}(\Omega)\right)$. However, $\widetilde{\nabla u_{\varepsilon}}$ can be shown to be a bounded sequence in $\left.L^{p}\left(\Omega_{T}\right)\right)$ and has a weak limit. We will improve the weak convergence through a corrector result. For this, we consider the solution $U_{1}(x, t, y) \in L^{p}\left(\Omega \times(0, T) ; W_{\text {per }}^{1, p}(Y)\right)$ of

$$
\int_{0}^{T} \int_{\Omega} \int_{Y \backslash S} a\left(y, \nabla_{x} u(x, t)+\nabla_{y} U_{1}(x, t, y)\right) \cdot \nabla_{y} \Phi(x, t, y) \mathrm{d} y \mathrm{~d} x \mathrm{~d} t=0
$$

for all $\Phi \in L^{p}\left(\Omega \times(0, T) ; W_{\text {per }}^{1, p}(Y)\right)$ (we will see that such a $U_{1}$ exists). Let $\chi$ be the characteristic function of $Y \backslash S$ defined on $Y$ and extended $Y$-periodically to $\mathbb{R}^{n}$. Then, $\chi(x / \varepsilon)$ is the characteristic function of $\Omega_{\varepsilon}$. The corrector result is as follows: 
Theorem 2.6. Let the functions $u_{\varepsilon}, u, U_{1}$ be as above. Assume further that (A5) holds and $u, U_{1}$ are sufficiently smooth, i.e. that they belong to $C^{1}(\Omega \times(0, T))$ and $C(\Omega \times$ $\left.(0, T) ; C_{\mathrm{per}}^{1}(Y)\right)$ respectively. Then

$$
\begin{aligned}
\tilde{u_{\varepsilon}}-u-\varepsilon U_{1}\left(x, t, \frac{x}{\varepsilon}\right) & \rightarrow 0 \text { strongly in } L^{p}\left(\Omega_{T}\right) \text { and }, \\
\widetilde{\nabla u_{\varepsilon}}-\chi\left(\frac{x}{\varepsilon}\right)\left(\nabla_{x} u+\nabla_{y} U_{1}\left(x, t, \frac{x}{\varepsilon}\right)\right) & \rightarrow 0 \text { strongly in } L^{p}\left(\Omega_{T}\right) .
\end{aligned}
$$

Remark 2.7. Note that, in particular, the $L^{p}$ weak limit of $\widetilde{\nabla u_{\varepsilon}}$ is $m^{*} \nabla u(x, t)+$ $\int_{\partial S} \frac{\partial U_{1}}{\partial v}(x, t, y) \mathrm{d} \sigma(y)$.

\section{Some preliminary results}

As we closely follow the treatment in [15], many results will only be sketched and we refer the reader to [15] for more details as and when necessary.

We first obtain a priori bounds under the assumption (2.10). From now on, $C$ will denote a generic positive constant which is independent of $\varepsilon$.

Lemma 3.1. Let $u_{\varepsilon}$ be a family of solutions of $\left(P_{\varepsilon}\right)$ and assume that (2.10) holds. Then,

$$
\begin{aligned}
\sup _{\varepsilon}\left\|\nabla u_{\varepsilon}\right\|_{L^{p}\left(\Omega_{\varepsilon} \times(0, T)\right)} & \leq C, \\
\sup _{\varepsilon}\left\|a\left(\frac{x}{\varepsilon}, \nabla u_{\varepsilon}\right)\right\|_{L^{p^{*}}\left(\Omega_{\varepsilon} \times(0, T)\right)} & \leq C, \\
\sup _{\varepsilon}\left\|\partial_{t} b\left(\frac{x}{\varepsilon}, u_{\varepsilon}\right)\right\|_{E_{\varepsilon}^{*}} & \leq C .
\end{aligned}
$$

Proof. Define the function $B(.,):. \mathbb{R}^{n} \times \mathbb{R} \rightarrow \mathbb{R}$ by

$$
B(y, s)=b(y, s) s-\int_{0}^{s} b(y, \tau) \mathrm{d} \tau .
$$

As in [15] we deduce that

$$
\begin{aligned}
& \int_{\Omega_{\varepsilon}} B\left(\frac{x}{\varepsilon}, u_{\varepsilon}(x, T)\right) \mathrm{d} x+\int_{0}^{T} \int_{\Omega_{\varepsilon}} a\left(\frac{x}{\varepsilon}, \nabla u_{\varepsilon}\right) \cdot \nabla u_{\varepsilon} \mathrm{d} x \mathrm{~d} t \\
& =\int_{\Omega_{\varepsilon}} B\left(\frac{x}{\varepsilon}, u_{0}\right) \mathrm{d} x+\int_{0}^{T} \int_{\Omega_{\varepsilon}} f u_{\varepsilon} \mathrm{d} x \mathrm{~d} t
\end{aligned}
$$

and from this we obtain

$$
\int_{\Omega} B\left(\frac{x}{\varepsilon}, u_{\varepsilon}(x, T)\right) \mathrm{d} x+\int_{0}^{T} \int_{\Omega_{\varepsilon}} a\left(\frac{x}{\varepsilon}, \nabla u_{\varepsilon}\right) \cdot \nabla u_{\varepsilon} \mathrm{d} x \mathrm{~d} t \leq C
$$

by (2.10) and the assumptions on $b$ and $a$. Then, (3.1) follows from (3.5) and (2.5), as $B$ is non-negative, while (3.2) follows from (3.1) and (2.7). The estimate (3.3) may be obtained from (3.1), (3.2) and (2.3). Thus the lemma.

We now show that $\tilde{u_{\varepsilon}} \rightarrow u$ a.e. $\Omega \times(0, T)$, for a subsequence and for some $u$. This is the crucial part of the present analysis. This result is to be seen in the context of the estimate 
(3.1) where there is no time gradient estimate. Hence, we cannot use any ready-made compact imbedding theorem to get the strong convergence of $u_{\varepsilon}$ to $u$ in some $L^{r}\left(\Omega_{T}\right)$. However, we are able to achieve this by adapting a technique found in [3]. This is given in the following lemma.

Lemma 3.2. Let $u_{\varepsilon}$ be as above. Then, the sequence $\left\{\tilde{u}_{\varepsilon}\right\}_{\varepsilon>0}$ is relatively compact in $L^{\theta}\left(\Omega_{T}\right)$, where $\theta$ is as in (A2). As a result, there is a subsequence of $u_{\varepsilon}$ such that,

$$
\tilde{u_{\varepsilon}} \rightarrow \text { u a.e. in } \Omega_{T} \text {. }
$$

Proof. Analogous to Step 1, Lemma 3.3 in [15], we can derive the following estimate

$$
\begin{array}{r}
h^{-1} \int_{0}^{T-h} \int_{\Omega_{\varepsilon}}\left(b\left(\frac{x}{\varepsilon}, u_{\varepsilon}(t+h)\right)-b\left(\frac{x}{\varepsilon}, u_{\varepsilon}(t)\right)\right)\left(u_{\varepsilon}(t+h)\right. \\
\left.-u_{\varepsilon}(t)\right) \mathrm{d} x \mathrm{~d} t \leq C
\end{array}
$$

for some constant $C$ which is independent of $\varepsilon$ and $h$. Thus, as we have assumed in (A1) that $b(y, 0)=0$, we get

$$
\begin{array}{r}
h^{-1} \int_{0}^{T-h} \int_{\Omega}\left(b\left(\frac{x}{\varepsilon}, \tilde{u_{\varepsilon}}(t+h)\right)-b\left(\frac{x}{\varepsilon}, \tilde{u_{\varepsilon}}(t)\right)\right)\left(\tilde{u_{\varepsilon}}(t+h)\right. \\
\left.-\tilde{u_{\varepsilon}}(t)\right) \mathrm{d} x \mathrm{~d} t \leq C .
\end{array}
$$

Proceeding as in [15] we obtain the desired result.

From Lemma 3.2 above, the continuity of $b$ and the assumption (2.10) we derive the following corollary.

\section{COROLLARY 3.3}

We have, $b\left(\frac{x}{\varepsilon}, \tilde{u_{\varepsilon}}\right)-b\left(\frac{x}{\varepsilon}, u\right) \rightarrow 0$ strongly in $L^{q}\left(\Omega_{T}\right) \forall q, 0<q<\infty$.

Proof. By the a priori bound (2.10), it is enough to consider the function $b$ on $Y \times[-M, M]$ for a large $M>0$. As $b$ is continuous, it is uniformly continuous on $Y \times[-M, M]$. Therefore, given $h_{0}>0$, there exists a $\delta>0$ such that,

$$
\left|b(y, s)-b\left(y^{\prime}, s^{\prime}\right)\right|<h_{0},
$$

whenever $\left|y-y^{\prime}\right|+\left|s-s^{\prime}\right|<\delta$.

Now, since $\tilde{u_{\varepsilon}} \rightarrow u$ a.e in $\Omega_{T}$, by Egoroff's theorem, given $h_{1}>0$, there exists $E \subset \Omega_{T}$ such that its Lebesgue measure $m(E)<h_{1}$ and $\tilde{u_{\varepsilon}}$ converges uniformly to $u$ on $\left(\Omega_{T} \backslash E\right)$, which we denote by $E^{\prime}$. Therefore, we can find $\varepsilon_{1}>0$ such that

$$
\left\|\tilde{u_{\varepsilon}}-u\right\|_{\infty, E^{\prime}}<\delta \forall \varepsilon<\varepsilon_{1} .
$$

Therefore, for $\varepsilon<\varepsilon_{1}$ we have

$$
\begin{aligned}
\int_{\Omega_{T}} & \left|b\left(\frac{x}{\varepsilon}, \tilde{u_{\varepsilon}}\right)-b\left(\frac{x}{\varepsilon}, u\right)\right|^{q} \mathrm{~d} x \mathrm{~d} t \\
= & \int_{E^{\prime}}\left|b\left(\frac{x}{\varepsilon}, \tilde{u_{\varepsilon}}\right)-b\left(\frac{x}{\varepsilon}, u\right)\right|^{q} \mathrm{~d} x \mathrm{~d} t \\
& +\int_{E}\left|\left(b\left(\frac{x}{\varepsilon}, \tilde{u_{\varepsilon}}\right)-b\left(\frac{x}{\varepsilon}, u\right)\right)\right|^{q} \mathrm{~d} x \mathrm{~d} t \\
\leq & h_{0}^{q} m\left(\Omega_{T}\right)+2^{q} \sup \left(|b|^{q}\right) m(E) \\
\leq & h_{0}^{q} m\left(\Omega_{T}\right)+2^{q} \sup \left(|b|^{q}\right) h_{1} .
\end{aligned}
$$


This completes the proof as $h_{0}$ and $h_{1}$ can be chosen arbitrarily small.

As a consequence, we have the following corollary.

\section{COROLLARY 3.4}

We have the following convergences:

$$
\begin{array}{r}
b\left(\frac{x}{\varepsilon}, \tilde{u_{\varepsilon}}\right) \rightarrow \bar{b}(u) \text { weakly in } L^{q}\left(\Omega_{T}\right), \\
\chi\left(\frac{x}{\varepsilon}\right) b\left(\frac{x}{\varepsilon}, \tilde{u_{\varepsilon}}\right) \rightarrow b^{*}(u) \text { weakly in } L^{q}\left(\Omega_{T}\right)
\end{array}
$$

for $q>1$. Further, $\bar{b}(u)=b^{*}(u)$.

Proof. We note that

$$
\begin{aligned}
b\left(\frac{x}{\varepsilon}, \tilde{u_{\varepsilon}}\right) & =\left(b\left(\frac{x}{\varepsilon}, \tilde{u_{\varepsilon}}\right)-b\left(\frac{x}{\varepsilon}, u\right)\right)+b\left(\frac{x}{\varepsilon}, u\right) \\
& \rightarrow 0+\bar{b}(u)
\end{aligned}
$$

by Corollary 3.3 and the averaging principle for periodic functions mentioned below.

Similarly,

$$
\begin{aligned}
\chi\left(\frac{x}{\varepsilon}\right) b\left(\frac{x}{\varepsilon}, \tilde{u_{\varepsilon}}\right) & =\chi\left(\frac{x}{\varepsilon}\right)\left(b\left(\frac{x}{\varepsilon}, \tilde{u_{\varepsilon}}\right)-b\left(\frac{x}{\varepsilon}, u\right)\right)+\chi\left(\frac{x}{\varepsilon}\right) b\left(\frac{x}{\varepsilon}, u\right) \\
& \rightarrow 0+b^{*}(u) .
\end{aligned}
$$

From $\chi\left(\frac{x}{\varepsilon}\right) b\left(\frac{x}{\varepsilon}, \tilde{u_{\varepsilon}}\right)=b\left(\frac{x}{\varepsilon}, \tilde{u_{\varepsilon}}\right)$, we readily obtain the last of the conclusions in the corollary.

The homogenization will be done in the framework of two-scale convergence. So to conclude this section, we recall an averaging principle which is at the basis of this method, the definition of two-scale convergence and a few related results which we will use in the sequel. For an exposition of this method and its main features, see $[14,1,15]$.

For $f(x, y) \in L_{\text {loc }}^{q}\left(\mathbb{R}^{n} ; C_{\mathrm{per}}(Y)\right)$, the oscillatory function $f\left(x, \frac{x}{\varepsilon}\right)$ converges weakly in $L_{\text {loc }}^{q}\left(\mathbb{R}^{n}\right)$ to $\int_{Y} f(x, y) \mathrm{d} y$, for all $q>1$. A similar result holds for $f(x, y) \in L_{\mathrm{loc}}^{\infty}\left(\mathbb{R}^{n} ; C_{\mathrm{per}}(Y)\right)$, with weak convergence replaced by weak-* convergence.

\section{DEFINITION 3.5}

Let $1<q<\infty$. A sequence of functions $v_{\varepsilon} \in L^{q}\left(\Omega_{T}\right)$ is said to two-scale converge to a function $v \in L^{q}\left(\Omega_{T} \times Y\right)$ if

$$
\int_{\Omega_{T}} v_{\varepsilon} \psi\left(x, t, \frac{x}{\varepsilon}\right) \mathrm{d} x \mathrm{~d} t \stackrel{\varepsilon \rightarrow 0}{\rightarrow} \int_{\Omega_{T}} \int_{Y} v(x, t, y) \psi(x, t, y) \mathrm{d} y \mathrm{~d} x \mathrm{~d} t
$$

for all $\psi \in L^{q^{*}}\left(\Omega_{T} ; C_{\mathrm{per}}(Y)\right)$. We write $v_{\varepsilon} \stackrel{2-s}{\rightarrow} v$.

Theorem 3.6. Let $1<q<\infty$ and let $v_{\varepsilon}$ be a bounded sequence in $L^{q}\left(\Omega_{T}\right)$. Then there exists a function $v \in L^{q}\left(\Omega_{T} \times Y\right)$ such that, up to a subsequence, $v_{\varepsilon} \stackrel{2-s}{\rightarrow} v(x, t, y)$. 
The following theorem [1] is useful in obtaining the limit of the product of two two-scale convergent sequences. Let $1<q<\infty$.

Theorem 3.7. Let $q>1$. Let $v_{\varepsilon}$ be a sequence in $L^{q}\left(\Omega_{T}\right)$ and $w_{\varepsilon}$ be a sequence in $L^{q^{*}}\left(\Omega_{T}\right)$ such that $v_{\varepsilon} \stackrel{2-s}{\rightarrow} v$ and $w_{\varepsilon} \stackrel{2-s}{\rightarrow} w$. Further, assume that the sequence $w_{\varepsilon}$ satisfies

$$
\int_{\Omega_{T}}\left|w_{\varepsilon}\right|^{q^{*}}(x, t) \mathrm{d} x \mathrm{~d} t \stackrel{\varepsilon \rightarrow 0}{\rightarrow} \int_{\Omega_{T}} \int_{Y}|w(x, t, y)|^{q^{*}} \mathrm{~d} y \mathrm{~d} x \mathrm{~d} t .
$$

Then,

$$
\int_{\Omega_{T}} v_{\varepsilon} w_{\varepsilon} \mathrm{d} x \mathrm{~d} t \stackrel{\varepsilon \rightarrow 0}{\rightarrow} \int_{\Omega_{T}} \int_{Y} v(x, t, y) w(x, t, y) \mathrm{d} y \mathrm{~d} x \mathrm{~d} t .
$$

Remark 3.8. A sequence $w_{\varepsilon}$ which two-scale converges and satisfies (3.8) is said to be strongly two-scale convergent. Examples of strongly two-scale convergent sequences are $\left\{\psi\left(x, t, \frac{x}{\varepsilon}\right)\right\}_{\varepsilon}$ for any $\psi \in L_{\mathrm{per}}^{q}\left(Y ; C\left(\Omega_{T}\right)\right)$.

We now state a result for perforated domains the proof of which may be found in [1].

Theorem 3.9. Let $q>1$. Let $v_{\varepsilon} \in L^{q}\left(\Omega_{\varepsilon} \times(0, T)\right)$ and let $\nabla v_{\varepsilon} \in L^{q}\left(\Omega_{\varepsilon} \times(0, T)\right)$ be such that

$$
\begin{gathered}
\sup _{\varepsilon}\left\|v_{\varepsilon}\right\|_{L^{q}\left(\Omega_{\varepsilon} \times(0, T)\right)} \leq C, \\
\sup _{\varepsilon}\left\|\nabla v_{\varepsilon}\right\|_{L^{q}\left(\Omega_{\varepsilon} \times(0, T)\right)} \leq C .
\end{gathered}
$$

Then there exist $v \in L^{q}\left(0, T ; W^{1, q}(\Omega)\right)$ and $V_{1} \in L^{q}\left(\Omega_{T} ; W_{\mathrm{per}}^{1, q}(Y)\right)$ such that, up to a subsequence

$$
\begin{aligned}
\widetilde{v_{\varepsilon}} & \stackrel{2-s}{\rightarrow} v(x, t), \\
\widetilde{\nabla v_{\varepsilon}} & \stackrel{2-s}{\rightarrow} \chi(y)\left(\nabla_{x} v(x, t)+\nabla_{y} V_{1}(x, t, y)\right) .
\end{aligned}
$$

\section{Proofs of the main Theorems}

We first obtain the two-scale homogenized equation. From the estimates (3.1) and (3.2) and Theorems 3.8 and 3.5, there exists $U_{1} \in L^{p}\left(\Omega_{T} ; W_{\text {per }}^{1, p}(Y)\right)$ and $a_{0} \in L^{p^{*}}\left(\Omega_{T} \times Y\right)$ such that, up to a subsequence,

$$
\begin{aligned}
& \widetilde{\nabla u_{\varepsilon}} \stackrel{2-s}{\rightarrow} \chi(y)\left(\nabla_{x} u(x, t)+\nabla_{y} U_{1}(x, t, y)\right), \\
& \chi\left(\frac{x}{\varepsilon}\right) a\left(\frac{x}{\varepsilon}, \widetilde{\nabla u_{\varepsilon}}\right) \stackrel{2-s}{\rightarrow} a_{0}(x, t, y) .
\end{aligned}
$$

Theorem 4.1. The limits $u, a_{0}$ satisfy the following two-scale homogenized problem

$$
\begin{aligned}
& \int_{0}^{T}\left\langle\partial_{t} b^{*}(u), \phi\right\rangle \mathrm{d} t+\int_{\Omega_{T}} \int_{Y \backslash S} a_{0}(x, t, y) \cdot\left(\nabla_{x} \phi+\nabla_{y} \Phi(x, t, y)\right) \mathrm{d} y \mathrm{~d} x \mathrm{~d} t \\
& =\int_{\Omega_{T}} f \phi \mathrm{d} x \mathrm{~d} t
\end{aligned}
$$

for all $\phi \in C_{0}^{\infty}\left(\Omega_{T}\right)$ and $\Phi \in C_{0}^{\infty}\left(\Omega_{T} ; C_{\mathrm{per}}^{\infty}(Y)\right)$. 
Proof. We pass to the limit in (2.3) in the presence of appropriate test functions. Let $\phi \in C_{0}^{\infty}\left(\Omega_{T}\right)$ and $\Phi \in C_{0}^{\infty}\left(\Omega_{T} ; C_{\text {per }}^{\infty}(Y)\right)$. Set,

$$
\phi_{\varepsilon}=\phi(x, t)+\varepsilon \Phi\left(x, t, \frac{x}{\varepsilon}\right) .
$$

We may use these as test functions in (2.3) as the restrictions of $\phi_{\varepsilon}$ to $\Omega_{\varepsilon}$ are in $E_{\varepsilon}$ and we note

$$
\begin{aligned}
\int_{0}^{T} & \left\langle\partial_{t} b\left(\frac{x}{\varepsilon}, u_{\varepsilon}\right), \phi_{\varepsilon}\right\rangle_{\varepsilon} \mathrm{d} t+\int_{0}^{T} \int_{\Omega} \chi\left(\frac{x}{\varepsilon}\right) a\left(\frac{x}{\varepsilon}, \widetilde{\nabla u_{\varepsilon}}\right) \\
\times & \left(\nabla_{x} \phi+\nabla_{y} \Phi\left(x, t, \frac{x}{\varepsilon}\right)\right) \mathrm{d} x \mathrm{~d} t+o(1)=\int_{0}^{T} \int_{\Omega} \chi\left(\frac{x}{\varepsilon}\right) f(x, t) \phi(x, t) \mathrm{d} x \mathrm{~d} t+o(1) .
\end{aligned}
$$

Now we pass to the limit in the above identity. We may handle the first term as follows. Observing that $\phi_{\varepsilon}(., T)=0=\phi_{\varepsilon}(., 0)$, we have from (2.2),

$$
\begin{aligned}
\int_{0}^{T}\left\langle\partial_{t} b\left(\frac{x}{\varepsilon}, u_{\varepsilon}\right), \phi_{\varepsilon}\right\rangle_{\varepsilon} \mathrm{d} t & =-\int_{0}^{T} \int_{\Omega_{\varepsilon}} b\left(\frac{x}{\varepsilon}, u_{\varepsilon}\right) \partial_{t} \phi_{\varepsilon} \mathrm{d} x \mathrm{~d} t \\
& =-\int_{0}^{T} \int_{\Omega} \chi\left(\frac{x}{\varepsilon}\right) b\left(\frac{x}{\varepsilon}, \tilde{u_{\varepsilon}}\right) \partial_{t} \phi \mathrm{d} x \mathrm{~d} t+o(1) \\
& \rightarrow-\int_{0}^{T} \int_{\Omega} b^{*}(u) \partial_{t} \phi \mathrm{d} x \mathrm{~d} t \\
& =\int_{0}^{T}\left\langle\partial_{t} b^{*}(u), \phi\right\rangle \mathrm{d} x \mathrm{~d} t,
\end{aligned}
$$

where the convergence in the above follows from Corollary 3.4.

For passing to the limit in the other two terms in (4.4) we observe that

$$
\begin{aligned}
& \int_{0}^{T} \int_{\Omega_{\varepsilon}} a\left(\frac{x}{\varepsilon}, \nabla u_{\varepsilon}\right) \cdot\left(\nabla_{x} \phi+\nabla_{y} \Phi\left(x, t, \frac{x}{\varepsilon}\right)\right) \mathrm{d} x \mathrm{~d} t \\
= & \int_{0}^{T} \int_{\Omega} \chi\left(\frac{x}{\varepsilon}\right) a\left(\frac{x}{\varepsilon}, \widetilde{\nabla u_{\varepsilon}}\right) \cdot\left(\nabla_{x} \phi+\nabla_{y} \Phi\left(x, t, \frac{x}{\varepsilon}\right)\right) \mathrm{d} x \mathrm{~d} t \\
\rightarrow & \int_{0}^{T} \int_{\Omega} \int_{Y} a_{0}(x, t, y) \cdot\left(\nabla_{x} \phi+\nabla_{y} \Phi(x, t, y)\right) \mathrm{d} y \mathrm{~d} x \mathrm{~d} t,
\end{aligned}
$$

where the convergence in the last step follows from Theorem 3.6. For this, one observes from Remark 3.7 that $\nabla_{x} \phi+\nabla_{y} \Phi\left(x, t, \frac{x}{\varepsilon}\right)$ is strongly two-scale convergent to $\nabla_{x} \phi+$ $\nabla_{y} \Phi(x, t, y)$. Further, observe that since $\chi\left(\frac{x}{\varepsilon}\right) a\left(\frac{x}{\varepsilon}, \widetilde{\nabla u_{\varepsilon}}\right)$ is zero in the holes, we have $a_{0}(x, t, y)$ to be zero in $S$ and the integral over $Y$ can be replaced by that over $Y \backslash S$.

Finally, note that

$$
\begin{aligned}
\int_{0}^{T} \int_{\Omega} \chi\left(\frac{x}{\varepsilon}\right) f(x, t) \phi(x, t) \mathrm{d} x \mathrm{~d} t & \rightarrow \int_{0}^{T} \int_{\Omega} \int_{Y} \chi(y) f(x, t) \phi(x, t) \mathrm{d} y \mathrm{~d} x \mathrm{~d} t \\
& =\int_{0}^{T} \int_{\Omega} m^{*} f \phi \mathrm{d} x \mathrm{~d} t .
\end{aligned}
$$

Thus, from the above we deduce that the limit of the equations (2.3) with $\phi_{\varepsilon}$ as test functions is nothing but (4.3). This is referred to as the two-scale homogenized problem. 
Remark 4.2. We note from the two-scale homogenized problem (4.3), by setting $\Phi \equiv 0$ that

$$
\partial_{t} b^{*}(u)-\operatorname{div}\left(\int_{Y \backslash S} a_{0}(x, t, y) \mathrm{d} y\right)=m^{*} f \text { in } \Omega \times(0, T)
$$

and by setting $\phi \equiv 0$ we get

$$
\int_{0}^{T} \int_{\Omega} \int_{Y \backslash S} a_{0}(x, t, y) \cdot \nabla_{y} \Phi(x, t, y) \mathrm{d} y \mathrm{~d} x \mathrm{~d} t=0
$$

for all $\Phi \in C_{0}^{\infty}\left(\Omega_{T} ; C_{\text {per }}^{\infty}(Y)\right)$. In view of (4.5) and (4.6), it is enough to show that $a_{0}(x, t, y)=a\left(y, \nabla_{x} u(x, t)+\nabla_{y} U_{1}(x, t, y)\right)$ to conclude Theorem 2.4.

Proof of Theorem 2.4. Note that (2.11) follows from the convergence (3.6) and the assumption (2.10). We now justify (2.12), for which it is enough to show that $a_{0}(x, t, y)$ equals $a\left(y, \nabla_{x} u(x, t)+\nabla_{y} U_{1}(x, t, y)\right)$, by the preceding remark.

Let $\xi \in L^{p}\left(0, T ; W_{0}^{1, p}(\Omega)\right) \cup W^{1,1}\left(0, T ; L^{\infty}(\Omega)\right)$ with $\xi(., T)=0=\xi(., 0)$. This may be used as a test function in (2.2) and we have

$$
\int_{0}^{T}\left\langle\partial_{t} b\left(\frac{x}{\varepsilon}, u_{\varepsilon}\right), \xi(x, t)\right\rangle_{\varepsilon} \mathrm{d} t+\int_{0}^{T} \int_{\Omega_{\varepsilon}} b\left(\frac{x}{\varepsilon}, u_{\varepsilon}\right) \partial_{t} \xi \mathrm{d} x \mathrm{~d} t=0 .
$$

This can be rewritten as

$$
\int_{0}^{T}\left\langle R_{\varepsilon}^{*} \partial_{t} b\left(\frac{x}{\varepsilon}, u_{\varepsilon}\right), \xi\right\rangle \mathrm{d} t+\int_{0}^{T} \int_{\Omega} \chi\left(\frac{x}{\varepsilon}\right) b\left(\frac{x}{\varepsilon}, \tilde{u_{\varepsilon}}\right) \partial_{t} \xi \mathrm{d} x \mathrm{~d} t=0 .
$$

Observe that $\sup _{\varepsilon}\left\|R_{\varepsilon}^{*} \partial_{t} b\left(\frac{x}{\varepsilon}, u_{\varepsilon}\right)\right\|_{L^{p^{*}}\left(0, T ; W^{-1, p^{*}(\Omega)}\right)} \leq C$ by (3.3) where $R_{\varepsilon}$ is the restriction operator from $L^{p}\left(0, T ; W_{0}^{1, p}(\Omega)\right)$ to $E_{\varepsilon}$. Thus for some $w \in L^{p^{*}}\left(0, T ; W^{-1, p^{*}}(\Omega)\right)$ and for a subsequence

$$
R_{\varepsilon}^{*} \partial_{t} b\left(\frac{x}{\varepsilon}, u_{\varepsilon}\right) \rightarrow w \text { weakly } * \text { in } L^{p^{*}}\left(0, T ; W^{-1, p^{*}}(\Omega)\right) .
$$

Thus, passing to the limit in (4.7) we get

$$
\int_{0}^{T}\langle w, \xi\rangle+\int_{0}^{T} \int_{\Omega} b^{*}(u) \partial_{t} \xi \mathrm{d} x \mathrm{~d} t=0
$$

for all $\xi$ as above. So, $\partial_{t} b^{*}(u)=w$ in the sense of distributions.

We now show that $a_{0}(x, t, y)=a\left(y, \nabla_{x} u(x, t)+\nabla_{y} U_{1}(x, t, y)\right)$. Let $\phi$, $\Phi$ be as in the proof of Theorem 4.1. Let $\lambda>0$ and $\psi \in C_{0}^{\infty}\left(\Omega_{T} ; C_{\text {per }}^{\infty}(Y)\right)^{n}$. Set

$$
\eta_{\varepsilon}(x, t) \doteq \nabla_{x} \phi(x, t)+\nabla_{y} \Phi\left(x, t, \frac{x}{\varepsilon}\right)+\lambda \psi\left(x, t, \frac{x}{\varepsilon}\right) .
$$

By (2.6) we note that

$$
\int_{0}^{T} \int_{\Omega_{\varepsilon}}\left(a\left(\frac{x}{\varepsilon}, \nabla u_{\varepsilon}\right)-a\left(\frac{x}{\varepsilon}, \eta_{\varepsilon}\right)\right) \cdot\left(\nabla u_{\varepsilon}-\eta_{\varepsilon}\right) \mathrm{d} x \mathrm{~d} t \geq 0 .
$$


We have

$$
\begin{aligned}
\int_{0}^{T} \int_{\Omega_{\varepsilon}} a\left(\frac{x}{\varepsilon}, \nabla u_{\varepsilon}\right) \cdot \nabla u_{\varepsilon} & =-\int_{0}^{T}\left\langle\partial_{t} b\left(\frac{x}{\varepsilon}, u_{\varepsilon}\right), u_{\varepsilon}\right\rangle \mathrm{d} t+\int_{0}^{T} \int_{\Omega_{\varepsilon}} f u_{\varepsilon} \mathrm{d} x \mathrm{~d} t \\
& \rightarrow-\int_{0}^{T}\left\langle\partial_{t} b^{*}(u), u\right\rangle+\int_{0}^{T} \int_{\Omega} m^{*} f u \mathrm{~d} x \mathrm{~d} t \\
& =\int_{0}^{T} \int_{\Omega} \int_{Y \backslash S} a_{0}(x, t, y) . \nabla_{x} u \mathrm{~d} y \mathrm{~d} x \mathrm{~d} t,
\end{aligned}
$$

where the last step follows from (4.5).

Also, since $\eta_{\varepsilon}$ and $a\left(\frac{x}{\varepsilon}, \eta_{\varepsilon}\right)$ are strongly two-scale convergent, Theorem 3.6 gives

$$
\begin{aligned}
& \int_{0}^{T} \int_{\Omega_{\varepsilon}} a\left(\frac{x}{\varepsilon}, \eta_{\varepsilon}\right) \cdot \nabla u_{\varepsilon} \mathrm{d} x \mathrm{~d} t=\int_{0}^{T} \int_{\Omega} a\left(\frac{x}{\varepsilon}, \eta_{\varepsilon}\right) \cdot \widetilde{\nabla u_{\varepsilon}} \mathrm{d} x \mathrm{~d} t \\
\rightarrow & \int_{0}^{T} \int_{\Omega} \int_{Y \backslash S} a\left(y, \nabla_{x} \phi+\nabla_{y} \Phi+\lambda \psi\right) .\left(\nabla_{x} u+\nabla_{y} U_{1}\right) \mathrm{d} y \mathrm{~d} x \mathrm{~d} t .
\end{aligned}
$$

Similarly,

$$
\begin{aligned}
& \int_{0}^{T} \int_{\Omega_{\varepsilon}} a\left(\frac{x}{\varepsilon}, \eta_{\varepsilon}\right) \cdot \eta_{\varepsilon} \mathrm{d} x \mathrm{~d} t=\int_{0}^{T} \int_{\Omega} \chi\left(\frac{x}{\varepsilon}\right) a\left(\frac{x}{\varepsilon}, \eta_{\varepsilon}\right) \cdot \eta_{\varepsilon} \mathrm{d} x \mathrm{~d} t \\
\rightarrow & \int_{0}^{T} \int_{\Omega} \int_{Y \backslash S} a\left(y, \nabla_{x} \phi+\nabla_{y} \Phi+\lambda \psi\right) \cdot\left(\nabla_{x} \phi+\nabla_{y} \Phi+\lambda \psi\right) \mathrm{d} y \mathrm{~d} x \mathrm{~d} t
\end{aligned}
$$

and,

$$
\begin{gathered}
\int_{0}^{T} \int_{\Omega_{\varepsilon}} a\left(\frac{x}{\varepsilon}, \nabla u_{\varepsilon}\right) \cdot \eta_{\varepsilon} \mathrm{d} x \mathrm{~d} t=\int_{0}^{T} \int_{\Omega} \chi\left(\frac{x}{\varepsilon}\right) a\left(\frac{x}{\varepsilon}, \widetilde{\nabla u_{\varepsilon}}\right) \cdot \eta_{\varepsilon} \mathrm{d} x \mathrm{~d} t \\
\rightarrow \int_{0}^{T} \int_{\Omega} \int_{Y \backslash S} a_{0}(x, t, y) \cdot\left(\nabla_{x} \phi+\nabla_{y} \Phi+\lambda \psi\right) \mathrm{d} y \mathrm{~d} x \mathrm{~d} t \\
=\int_{0}^{T} \int_{\Omega} \int_{Y \backslash S} a_{0}(x, t, y) \cdot\left(\nabla_{x} \phi+\lambda \psi\right) \mathrm{d} y \mathrm{~d} x \mathrm{~d} t .
\end{gathered}
$$

The equality in the last step follows from (4.6). Thus

$$
\begin{aligned}
0 & \leq \lim _{\varepsilon \rightarrow 0} \int_{0}^{T} \int_{\Omega_{\varepsilon}}\left(a\left(\frac{x}{\varepsilon}, \nabla u_{\varepsilon}\right)-a\left(\frac{x}{\varepsilon}, \eta_{\varepsilon}\right)\right) \cdot\left(\nabla u_{\varepsilon}-\eta_{\varepsilon}\right)=\int_{0}^{T} \int_{\Omega} \int_{Y \backslash S} a_{0}(x, t, y) \\
& \times\left(\nabla_{x}(u-\phi)-\lambda \psi\right)-\int_{0}^{T} \int_{\Omega} \int_{Y \backslash S} a\left(y, \nabla_{x} \phi+\nabla_{y} \Phi+\lambda \psi\right) \\
& \times\left(\nabla_{x}(u-\phi)+\nabla_{y}\left(U_{1}-\Phi\right)-\lambda \psi\right) .
\end{aligned}
$$

Letting $\phi \rightarrow u$ and $\Phi \rightarrow U_{1}$ in the above and using the continuity of $a$ we get

$$
\int_{0}^{T} \int_{\Omega} \int_{Y \backslash S}\left(a\left(y, \nabla_{x} u+\nabla_{y} U_{1}+\lambda \psi\right)-a_{0}(x, t, y)\right) . \lambda \psi \mathrm{d} y \mathrm{~d} x \mathrm{~d} t \geq 0
$$


for all $\lambda$ and $\psi$. Dividing the above inequality by $\lambda$ and letting $\lambda \rightarrow 0$ we get,

$$
\int_{0}^{T} \int_{\Omega} \int_{Y \backslash S}\left(a\left(y, \nabla_{x} u+\nabla_{y} U_{1}\right)-a_{0}(x, t, y)\right) \cdot \psi \mathrm{d} y \mathrm{~d} x \mathrm{~d} t \geq 0
$$

for all $\psi$. From this we conclude that $a_{0}(x, t, y)=a\left(y, \nabla_{x} u+\nabla_{y} U_{1}\right)$.

Proof of Theorem 2.6. Set

$$
\eta_{\varepsilon}(x, t) \doteq \nabla_{x} u(x, t)+\nabla_{y} U_{1}\left(x, t, \frac{x}{\varepsilon}\right) .
$$

From (2.9) it follows that

$$
\begin{aligned}
\int_{0}^{T} \int_{\Omega_{\varepsilon}}\left(a\left(\frac{x}{\varepsilon}, \nabla u_{\varepsilon}\right)\right. & \left.-a\left(\frac{x}{\varepsilon}, \eta_{\varepsilon}\right)\right) \cdot\left(\nabla u_{\varepsilon}-\eta_{\varepsilon}\right) \mathrm{d} x, \mathrm{~d} t \\
& \geq \alpha \int_{0}^{T} \int_{\Omega_{\varepsilon}}\left|\nabla u_{\varepsilon}-\eta_{\varepsilon}\right|^{p} \mathrm{~d} x \mathrm{~d} t
\end{aligned}
$$

that is,

$$
\begin{aligned}
& \int_{0}^{T} \int_{\Omega_{\varepsilon}}\left(a\left(\frac{x}{\varepsilon}, \widetilde{\nabla u_{\varepsilon}}\right)-a\left(\frac{x}{\varepsilon}, \eta_{\varepsilon}\right)\right) \cdot\left(\widetilde{\nabla u_{\varepsilon}}-\chi\left(\frac{x}{\varepsilon}\right) \eta_{\varepsilon}\right) \mathrm{d} x \mathrm{~d} t \\
& \geq \alpha \int_{0}^{T} \int_{\Omega}\left|\nabla \widetilde{u_{\varepsilon}}-\chi\left(\frac{x}{\varepsilon}\right)\left[\nabla_{x} u(x, t)+\nabla_{y} U_{1}\left(x, t, \frac{x}{\varepsilon}\right)\right]\right|^{p} \mathrm{~d} x \mathrm{~d} t .
\end{aligned}
$$

As we have assumed that $u, U_{1}$ are smooth, we may proceed as in the proof of Theorem 2.4 to obtain

$$
\begin{gathered}
\alpha \underset{\varepsilon \rightarrow 0}{\lim \sup _{\varepsilon}} \int_{0}^{T} \int_{\Omega}\left|\nabla \tilde{u_{\varepsilon}}-\chi\left(\frac{x}{\varepsilon}\right)\left(\nabla_{x} u(x, t)+\nabla_{y} U_{1}\left(x, t, \frac{x}{\varepsilon}\right)\right)\right|^{p} \mathrm{~d} x \mathrm{~d} t \\
\leq \lim _{\varepsilon \rightarrow 0} \int_{0}^{T} \int_{\Omega_{\varepsilon}}\left(a\left(\frac{x}{\varepsilon}, \widetilde{\nabla u_{\varepsilon}}\right)-a\left(\frac{x}{\varepsilon}, \eta_{\varepsilon}\right)\right) \cdot\left(\widetilde{\nabla u_{\varepsilon}}-\chi\left(\frac{x}{\varepsilon}\right) \eta_{\varepsilon}\right) \mathrm{d} x \mathrm{~d} t \\
=0 .
\end{gathered}
$$

This completes the proof.

Remark 4.3. A weaker form of the corrector result with no smoothness assumptions can also be obtained. See [15] for the corresponding formulation.

\section{Acknowledgement}

The second author would like to thank the National Board of Higher Mathematics, India for financial support. He would also like to thank the organizers for their invitation to give a talk at the Workshop on 'Spectral and Inverse Spectral Theories for Schrödinger Operators' held at Goa, where he presented this work. 


\section{References}

[1] Allaire G, Homogenization and two-scale convergence, SIAM J. Math. Anal. 23 (1992) 1482-1518.

[2] Allaire G, Murat M and Nandakumar A K, Appendix of 'Homogenization of the Neumann problem with nonisolated holes', Asymptotic Anal. 7(2) (1993) 81-95

[3] Alt H W and Luckhaus S, Quasilinear elliptic-parabolic differential equations, Math. Z. 183 (1983) 311-341

[4] Avellaneda M and Lin F H, Compactness methods in the theory of homogenization, Comm. Pure Appl. Math. 40 (1987) 803-847

[5] Bensoussan B, Lions J L and Papanicolaou G, Asymptotic Analysis of Periodic Structures (Amsterdam: North Holland) (1978)

[6] Brahim-Omsmane S, Francfort G A and Murat F, Correctors for the homogenization of wave and heat equations, J. Math. Pures Appl. 71 (1992) 197-231

[7] Clark G W and Showalter R E, Two-scale convergence of a model for flow in a partially fissured medium, Electron. J. Differ. Equ. 1999(2) (1999) 1-20

[8] Douglas Jr. J, Peszyńska M and Showalter R E, Single phase flow in partially fissured media, Trans. Porous Media 28 (1995) 285-306

[9] Fusco N and Moscariello G, On the homogenization of quasilinear divergence structure operators, Ann. Mat. Pura Appl. 146(4) (1987) 1-13

[10] Hornung U, Applications of the homogenization method to flow and transport in porous media, Notes of Tshingua Summer School on Math. Modelling of Flow and Transport in Porous Media (ed.) Xiao Shutie (Singapore: World Scientific) (1992) pp. 167-222

[11] Jian H, On the homogenization of degenerate parabolic equations, Acta Math. Appl. Sinica 16(1) (2000) 100-110

[12] Jikov V V, Kozlov S M and Oleinik O A, Homogeniztion of Differential Operators and Integral Functionals (Berlin: Springer-Verlag) (1994)

[13] Ladyzenskaya O A, Solonnikov V and Ural'tzeva N N, Linear and quasilinear equations of parabolic type, Am. Math. Soc. Transl. Mono.? 23, (1968)

[14] Nguetseng G, A general convergence result of a functional related to the theory of homogenization, SIAM J. Math. Anal. 20 (1989) 608-623

[15] Nandakumaran A K and Rajesh M, Homogenization of a nonlinear degenerate parabolic differential equation, Electron. J. Differ. Equ. 2001(17) (2001) 1-19

[16] Oleinik O A, Kozlov S M and Zhikov V V, On $G$-convergence of parabolic operators, Ouspekhi Math. Naut. 36(1) (1981) 11-58 